\title{
A survey on Applying Ontological Engineering Approach for Hepatobiliary System Diseases
}

\author{
Galal AL Marzoqi, Marco Alfonse, Ibrahim F. Moawad, Mohamed Roushdy \\ Faculty of Computer and Information Science, Ain shams University, Abbasia, Cairo, Egypt \\ galalalmarzoqi@gmail.com, marco@fcis.asu.edu.eg, ibrahim_moawad@cis.asu.edu.eg, mroushdy@cis.asu.edu.eg
}

\begin{abstract}
Medical Ontologies play a central role in integrating heterogeneous databases of various model organisms. Hepatobiliary system is very important to human vital processes. It has an ability to regulate the other systems. Furthermore, it may be affected by many pathologic conditions, which affect other organs negatively. This paper investigates the current studies on Ontological engineering approach and Ontology techniques for Hepatobiliary System Diseases. We present conceptual view for the Hepatobiliary system and its infected diseases. Besides, we propose a new classification schema for the research efforts investigated so far. We classified the research efforts investigated so far based on the Hepatobiliary system organs: Liver, Gallbladder, Bile duct and Pancreas. Besides, we discuss the current research gaps found in this research area.
\end{abstract}

Keywords—Hepatobiliary System Diseases; Ontology Engineering; Protégé; Medical Systems;

\section{INTRODUCTION}

Ontology is a kind of controlled vocabulary of well defined terms with specified relationships between those terms, capable of interpretation by both humans and computers [1]. Furthermore, it is a specific rich description of Terminology, Rules, Concepts, and Relations among the concepts. There are several Ontology languages such as Extensible Markup Language (XML) [2], Resource Description Framework Schema (RDF(S)) [3], Darpa Agent Markup Language Ontology Interface Language (DAML+OIL) [4], and Web Ontology Language (OWL) [5]. The Ontologies can be exploited in many applications in fields, where semantics-based communication among people and systems are crucial. [6]. There are different techniques related to the Ontologies: Ontology alignment, Ontology mapping/matching, Ontology translation, Ontology merging/integrating Ontology refinement and Ontology unification [7].

Ontology tools can be applied for all stages of the Ontology lifecycle (creation, population, validation, deployment, maintenance, and evolution), and hence there are many tools for Ontologies management in different formats (Protégé, OilEd, Apollo, RDFedt, OntoLingua, OntoEdit, WebODE, KAON, ICOM, DOE, and WebOnto) [8]. Ontology can be used to support various knowledge management issues including knowledge retrieval, storing, and sharing [9]. Protégé is an open source software that provides user community with a suite of tools to construct domain models and knowledge-based applications with Ontologies Protégé implements a rich set of knowledge-modeling structures and actions that support the creation, visualization, and manipulation of Ontologies in various representation formats. Protégé can be customized to provide domain for creating knowledge models and entering data [10]. Medical Ontologies play a central role in integrating heterogeneous databases of various model organisms and

stored in heterogeneous databases. Also, it defines a precise and shared vocabulary for the semantic markup of resources and their description by metadata [11]. Conceptually, it is interested in solving important issues such as the reusing and sharing of medical data. The unambiguous communication of complex and detailed medical concepts is now a crucial feature of medical information systems [12]. There are Medical Ontologies developed to facilitate this purpose such as (Open Biomedical Ontologies OBO [13] , National Center for Biomedical Ontology NCBO's BioPortal [14] and Unified Medical Language System UMLS [15] ). Medical Ontology is now widely acknowledged that Ontologies can make a significant contribution to the design and implementation of information systems in the medical field.

On the other hand, human body systems consist of specific cells, tissues, and organs that work together to perform specific functions. Conceptually, these systems are interconnected and dependent so they can't work separately such as (Nervous System, Respiratory System, Immune System, Digestive System and Hepatobiliary System) [16,17]. The Hepatobiliary system includes four organs (Liver, Gallbladder, Bile duct and Pancreas). Where, Hepatobiliary is the one of the important systems in human body. It is responsible for lots of processes inside the body. These processes are important to keep body regulated and healthy. Conceptually, it plays an important role in many body functions like protein production. It is also responsible for detoxification, metabolism, synthesis, and storage of various substances however, this vital system may be affected by hazardous conditions whether they are internal or external [18, 
19]. Interestingly, these diseases were classified based on different dimensions such as (Cause, Treatment, Symptoms, etc). This paper presents a new classification schema for the research efforts investigated so far. We classified the research efforts investigated so far based on the Hepatobiliary system organs: Liver, Gallbladder, Bile duct and Pancreas.

The paper is organized as follows. Section 2 presents the conceptual view of Ontology based Hepatobiliary Systems. Section 3 displays Liver based Systems, section 4 presents Gallbladder based System, section 5 presents Pancreas based Systems, and section 6 presents Comparative among Ontology based systems for Hepatobiliary system diseases. Finally, section 7 presents conclusion and future work.

\section{CONCEPTUAL VIEW ONTOLOGY BASED HEPATOBILIARY SYSTEMS}

In general human body consists of many systems such as (Nervous System, Respiratory System, Immune System, Digestive System and Hepatobiliary System). The Hepatobiliary system includes four organs (Liver, Gallbladder, Bile duct and Pancreas). To understand the research paper so far Hepatobiliary system, we design conceptual view for human body and its related systems. Figure 1 shows a new classification schema in the "Human Body" is the main class. Also we have the "system" class. The "system" class into six subclasses which are "Nervous", "Respiratory", "Immune", "Digestive", "Hepatobiliary" and "Others". The "Hepatobiliary" class has "Organ" class. The "Organ" class includes five subclasses: "Liver", "Gallbladder", "Bile duct", "Pancreas" and "Disease". The "Disease" class includes eight instances: "Viral Hepatitis", "Liver Cancer", "Liver Immune", "Chronic", "Diabetes", "Anti-diabetic drugs", "HepatoPancreato-biliary" and "Lithiasic Cholecystitis" which are effect on organs: Liver, Gallbladder, Bile duct and Pancreas in Hepatobiliary system.

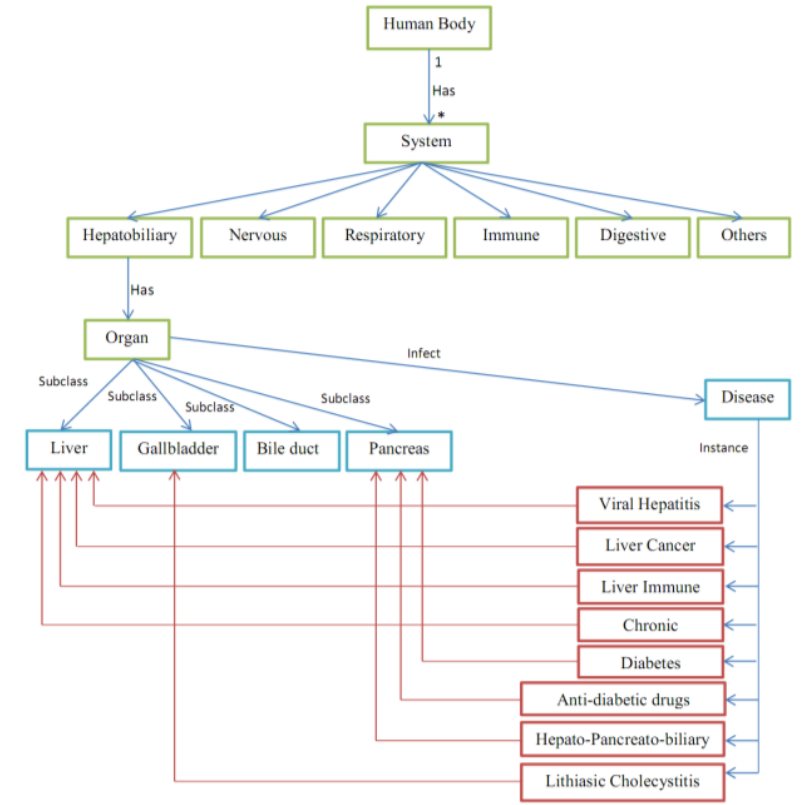

Fig.1. Conceptual view of Hepatobiliary System

There are many domains specific Ontological engineering approach and Ontology techniques has been built on Hepatobiliary system diseases. Figure 2 shows the research efforts investigated so far based on the Hepatobiliary system organs: Liver, Gallbladder, Bile duct and Pancreas.

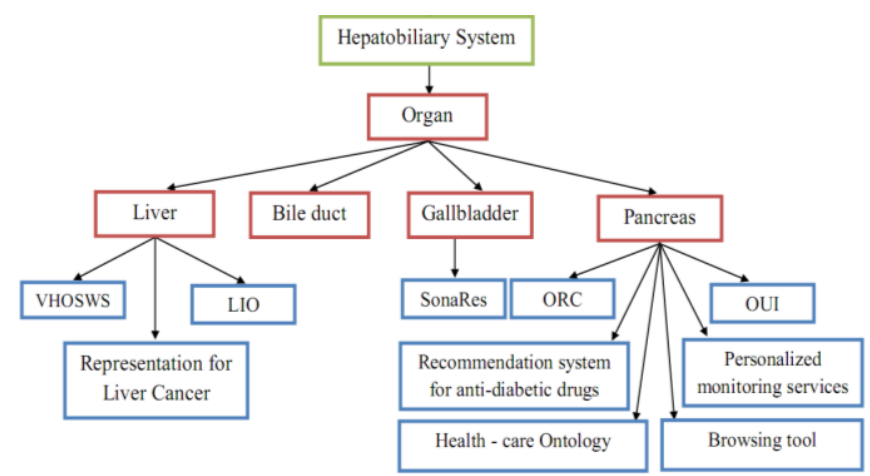

Fig.2. Ontology Based Systems for Hepatobiliary System Diseases

\section{LIVER BASED SYSTEMS}

In [20], the authors presented a web service based approach to share the Viral Hepatitis Ontology among physicians, students of medicine, and intelligent systems. In addition, the proposed approach enables physicians, and students of medicine to differentially diagnose the Viral Hepatitis diseases. To show how the approach is very beneficial for physicians and students of medicine, the authors developed a system prototype (VHOSWS) to present different usage case studies. In figure 3 the VHOSWS tool for Viral Hepatitis differential diagnosis is shown. 


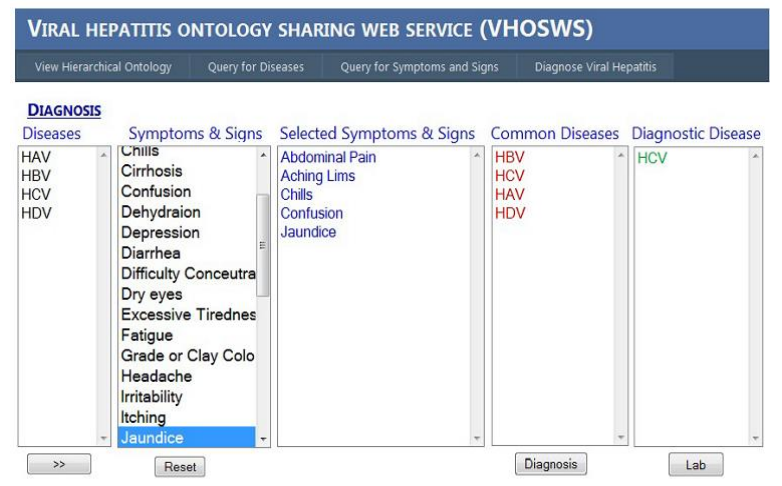

Fig.3. Viral Hepatitis Differential Diagnosis [20]

In [21], the authors developed Ontology based knowledge representation for Liver Cancer that was built using the Protégé-OWL editing environment. It has a great user interface that eases the process of building or editing Ontologies. This Ontology is encoded in OWL-DL format which is the most recent development in standard Ontology languages, endorsed by the World Wide Web Consortium (W3C) to promote the Semantic Web vision.

This Ontology can be used by experts or medical researchers who want the liver cancer knowledge to be represented in a semantic way that allows reasoning capabilities. Figure 4 shows the liver cancer class hierarchy.

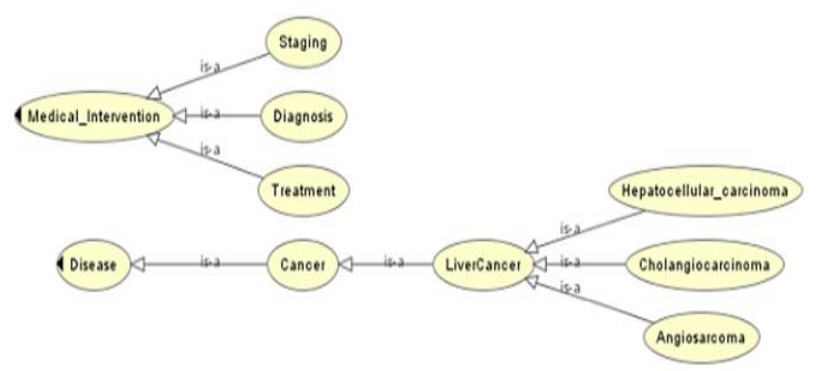

Fig.4. Liver Cancer class hierarchy [adapted from [21]]

In [22], the authors developed Liver Immunology Ontology (LIO) within the Open Biomedical Ontologies (OBO) Foundry framework, importing and linking relevant portions of orthogonal reference Ontologies. LIO is a novel tool for comprehensive analysis of liver immunology data sets, providing a valuable resource for the liver disease research community.

\section{GALLBLADDER BASED SYSTEM}

In [23], the authors presented description of the process of Ontology construction for gallbladder ultrasound images. This Ontology is inspired and based on the knowledge base created and being used for SonaRes the decision support system for ultrasound diagnostics. This system has accumulated the experience of the skillful experts-sonographists in the domain of hepato-pancreato-biliary zone examination. This experience and knowledge is well structured and formalized in this system for gallbladder and pancreas.
On the other hand, there is a powerful and attractive, from the point of view of knowledge portability, tool- Ontology, which in computer science is considered as an attempt of comprehensive and detailed formalization of some knowledge domain with the help of conceptual scheme. In figure 5 represents of knowledge on gallbladder pathology.

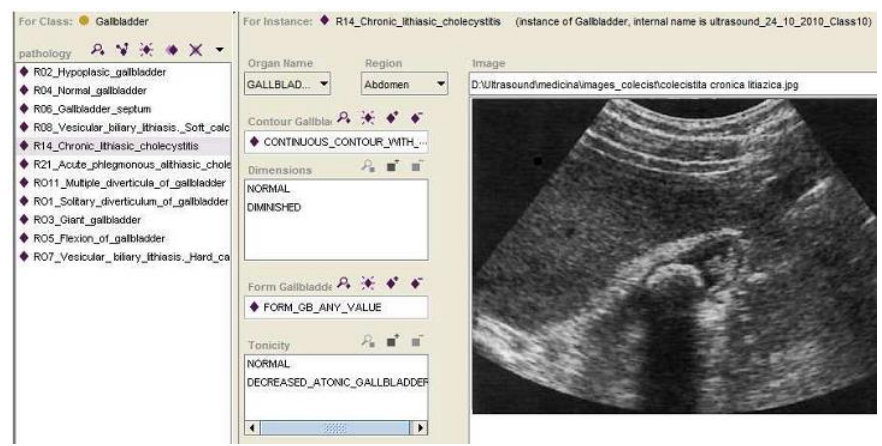

Fig.5. Knowledge on gallbladder pathology"Chronic lithiasic cholecystitis" [23]

\section{PANCREAS BASED SYSTEMS}

In [24], the authors presented a method of context-driven annotation in images of the DICOM standard [25] and its application for ultrasound images. Here is described them attempt to create a mapping between the Classification of Diseases ICD-10 [26], and the Ontology of Ultrasound Images (OUI) of hepato-pancreato-biliary zone organs [27].

In [28], the authors developed a disease Ontology based on River Flow Model and a browsing tool for causal chains defined in it. Because the Ontology is based on Ontological consideration of causal chains, it could capture characteristics of diseases appropriately. The definition of disease as causal could be also very friendly to clinicians since it is similar to their understanding of disease in practice. Moreover, it could include richer information about causal relationships in disease than other disease Ontologies or medical terminologies such as SNOMED-CT. Figure 6 presents the types of diabetes constituted of casual chain.

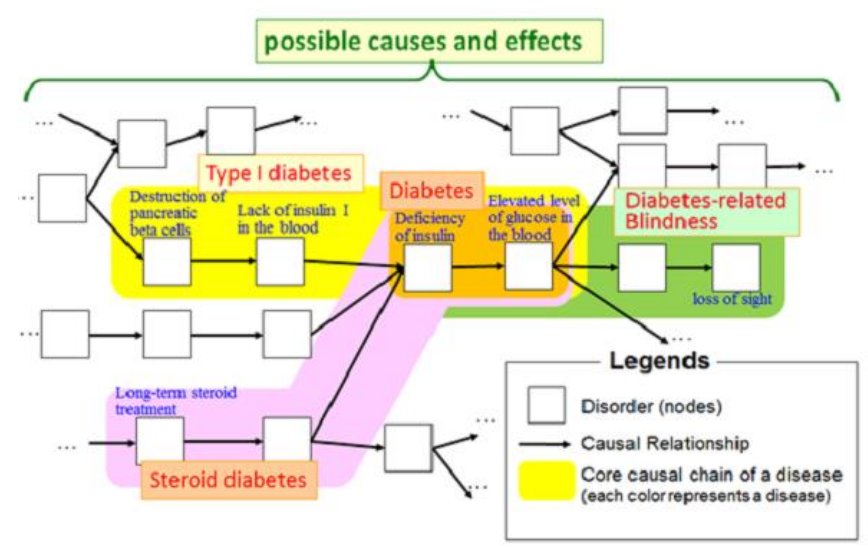


Fig.6. Types of Diabetes constituted of casual chain [28]

In [29], the authors presented an Ontology reasoning component (ORC) that builds upon existing Ontology modeling tools and techniques to support the integration and interpretation of multimodal medical information. $\mathrm{He}$ had illustrated how to embed ORC as a reasoning capability in reactive infrastructure agents supporting intelligent agents operating in COMMODITY ${ }_{12}$, a personal health environment for diabetic patients and the medical professionals that treat them. In figure 7 presents architecture showing how to extend the COMMODITY 12 PHS [30], with Ontologies and ORC agents to support semantic reasoning for diabetes patient profiles.

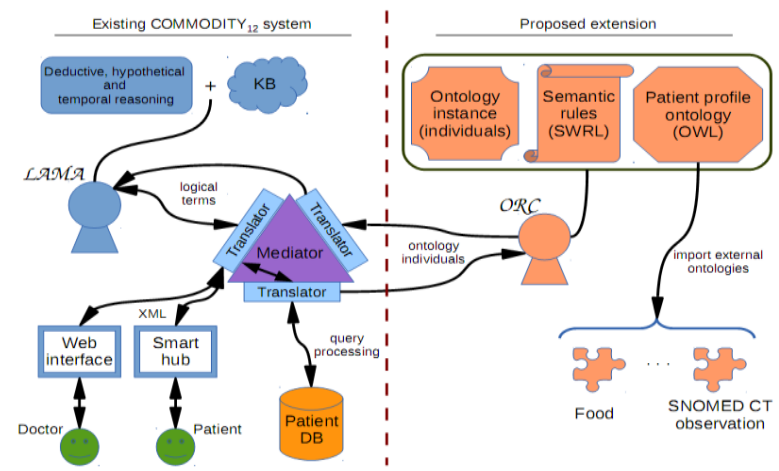

Fig.7. Architecture showing how to extend the COMMODITY12 PHS with Ontologies [29]

In [31], the authors developed a Diabetes Medication Recommendation system, based on domain Ontology that employ the knowledge base provided by a hospital specialist in Taichung's Department of Health and the database of the American Association of Clinical Endocrinologists Medical Guidelines for Clinical Practice for the Management of Diabetes Mellitus (AACEMG). By thorough analysis, the system first builds ontology knowledge about the drugs' nature attributes, type of dispensing and side effects, and ontology knowledge about patients' symptoms. It then utilizes Semantic Web Rule Language (SWRL) and Java Expert System Shell (JESS) to induce potential prescriptions for the patients. This system is able to analyze the symptoms of diabetes as well as to select the most appropriate drug from related drugs.

In [32], the authors purposed is to offer through three simple stages a solution based on Ontologies to provide personalized monitoring services for patients with any of a wide range of chronic conditions in a tele-monitoring scenario. Presenting the work through the three stages, actions involved in each stage are clearly described enhancing its understanding, reusability and transferability of both the Ontology and the methodology for different domains or applications.

In [33], the authors developed Ontology for the care of chronically ill patients and implement two personalization processes and a decision support tool. The first personalization process adapts the contents of the Ontology to the particularities observed in the health-care record of a given concrete patient, automatically providing a personalized Ontology containing only the clinical information that is relevant for health-care professionals to manage that patient. The second personalization process uses the personalized Ontology of a patient to automatically transform intervention plans describing health-care general treatments in to individual intervention plans. For comorbid patients, this process concludes with the semi-automatic integration of several individual plans in to a single personalized plan.

Finally, the ontology is also used as the knowledge base of a decision support tool that helps health-care professionals to detect anomalous circumstances such as wrong diagnoses, unobserved comorbidities, missing information, unobserved related diseases, or preventive actions.

\section{COMPARATIVE AMONG ONTOLOGY BASED SYSTEM FOR HEPATOBILIARY SYSTEM DISEASES}

There are many research works described different types of diseases and Ontological engineering approach on Hepatobiliary system. These works have been achieved to build specific domain Ontologies and systems for different diseases in Hepatobiliary system as shown in table1.

Table1: Represents comparison among Ontology-based systems for Hepatobiliary System Diseases

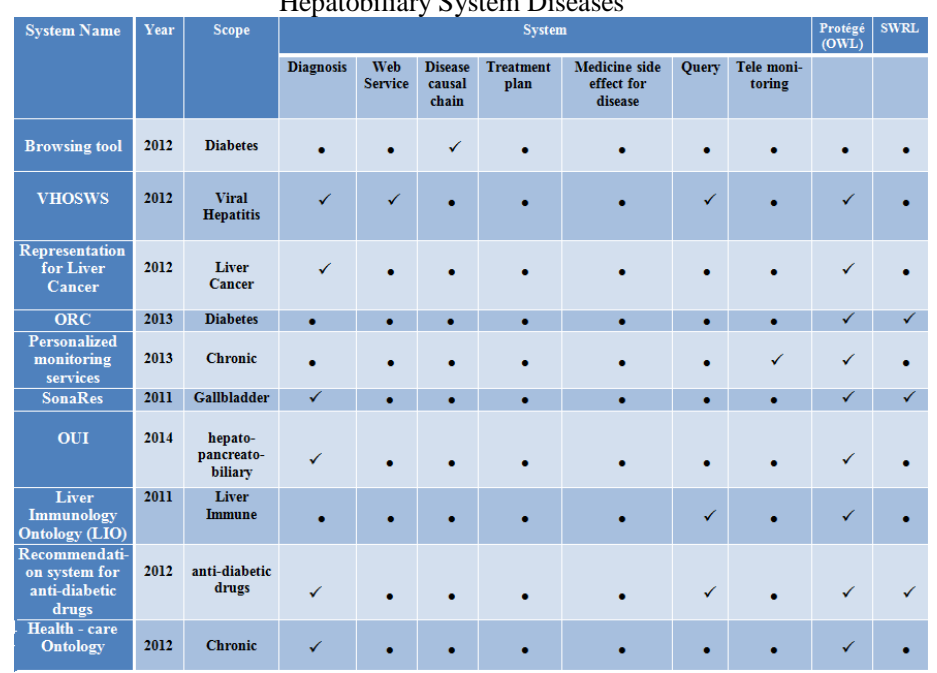

Browsing tool is system for disease casual chain in diabetes. VHOSWS is web service system for viral hepatitis diagnosis and query by using Ontology which is built by protégé editor (OWL file). Representation for Liver Cancer is system for liver cancer diagnosis by using Ontology which is built by protégé editor (OWL file). Ontology reasoning component (ORC) is Ontology in diabetes field which is built by protégé editor (OWL file) using Semantic Web Rule 
Language (SWRL). A personalized monitoring service is system for chronic tele- monitoring which is built by protégé editor (OWL file). While, SonaRes is system for Gallbladder diagnosis which is built by protégé editor (OWL file). Ontology of Ultrasound Images (OUI) is system for hepatopancreato-biliary zone organs diagnosis which is built by protégé editor (OWL file). Liver Immunology Ontology (LIO) is system for LIO query which is built by protégé editor (OWL file). Recommendation system based for anti-diabetic drugs diagnosis and query which is built by protégé editor (OWL file) using SWRL. On the other hand, health-care Ontology is system for chronic diagnosis which is built by protégé editor (OWL file).

\section{CONCLUSION AND FUTURE WORK}

This paper discussed the current studies on Ontological engineering approach and Ontology techniques for Hepatobiliary system diseases. It presented conceptual view of Ontology based Hepatobiliary system and its infected diseases. Furthermore, it presented a proposed a new classification schema for the research efforts investigated so far based on the Hepatobiliary system organs: Liver, Gallbladder, Bile duct and Pancreas. Beside, paper shows researchers worked in vary systems and Ontological engineering approach on Hepatobiliary system. The current work builds a new Ontology which using protégé editor (OWL file) and system for diagnosis, disease causal relation and query. Additionally, medicine side effects for disease, treatment plan and electronic patient records (EPRs) on different types of diseases in Hepatobiliary system.

\section{REFERENCES}

[1] Cicortas, A., Iordan, V., Fortis, A,'Considerations on Construction Ontologies", Journal Annals Computer Science Series 1, 79-88, 2009 .

[2] W3C XML Schema Definition Language, http://www.w3.org/TR/xmlschema11-1/, last visited 12 Oct, 2014

[3] Resource Description Framework (RDF) Schema Specification. http://www.w3.org/TR/PR-rdf-schema/ , last visited 29 Oct, 2014

[4] DAML+OIL Web Ontology Language. http://www.w3.org/Submission/2001/12/ , last visited 29 Oct, 2014.

[5] Web Ontology Language (OWL) Overview. http://www.w3.org/TR/owl-features/, last visited 29 Oct, 2004.

[6] Zhanjun Li, Maria C. Yang and Karthik Ramani, "A methodology for engineering ontology Acquisition and validation", Artificial Intelligence for Engineering Design, Analysis and Manufacturing, PP: 37-51, USA, 2009

[7] Moise Gabriela, Netedu Loredana," Ontologies for Interoperability in the eLearning Systems", Bulletin of Petroleum-Gas University of Ploiesti Mathematics, Informatics, Physics Series [BMIF], ISSN 1224-4899, EISSN 2067-242X, Volume LXI No. 2, PP: 75-88, 2009.

[8] Youn Seongwook, Arora Anchit, Chandrasekhar Preetham, Jayanty Paavany, Mestry Ashish and Sethi Shikha. "Survey about Ontology Development Tools for Ontology-based Knowledge Management", University of Southern California, 2009.

[9] H. Pundt and Y. Bishr. "Domain Ontologies for Data Sharing-An Example from Environmental Monitoring Using Field GIS", Computer \& Geosciences, 28, PP: 98-102, 1999.

[10] Protégé. http://protege.stanford.edu/ , last visited 29 Oct, 2012.
[11] Manolis Maragoudakis and Ilias Maglogiannis, "A medical ontology for intelligent web-based skin lesions image retrieval", Journal Health Informatics Journal, PP: 140-157, 2011.

[12] Sánchez, D., Moreno, A. "Learning Medical Ontologies from the Web", LNCS Knowledge management for Health care Procedures, Vol. 4924, PP: 32-45, 2008.

[13] Smith B, Ceusters W, Klagges B, Köhler J, Kumar A, Lomax J, Mungall C, Neuhaus F, Rector AL \& Rosse C, "Relations in biomedical ontologies", Genome Biology, Vol. 6, 2005.

[14] Samantha Bail, Matthew Horridge, Bijan Parsia, Ulrike Sattler, "The Justificatory Structure of the NCBO BioPortal Ontologies", Proceeding of The Semantic Web ISWC, Vol. 7031, PP: 67-82, 2011.

[15] C. Paul Morrey, James Geller, Michael Halper \& Yehoshua Perl, "The neighborhood auditing tool: A hybrid interface for auditing the UMLS", Journal of biomedical informatics, Vol. 42, PP: 468 489, 2009.

[16] Mythili Thirugnanam, Mangayarkarasi Ramaiah, V Pattabiraman, R Sivakumar, "Ontology based Disease Information System", International Conference on Modelling Optimization and Computing, PP: 3235- 3241, 2012.

[17] Official Partner of the Liver Strong Foundation, http://www.livestrong.com/article/119869-list-body-systems , last visited 10 December, 2014

[18] Medicine

Net, http://www.medicinenet.com/liver_disease/article.htm/ , last visited 10 December, 2014.

[19] http://hepatitis.about.com/od/jkl/g/liver.htm/ , last visited 10 December, 2014

[20] Moawad,I, Al Marzoqi,G, Salem, A," Web Service Based Approach for Viral Hepatitis Ontology Sharing and Diagnosing”, In Proceeding of AMLTA, PP: 257-266, Springer-Verlag Berlin Heidelberg 2012.

[21] Marco Alfonse, Mostafa M. Aref, Abdel-Badeeh M. Salem. "Ontology-Based Knowledge Representation for Liver Cancer". Proceedings of the International eHealth, Telemedicine and Health ICT Forum for Educational, Networking and Business. Luxembourg, G. D. of Luxembourg, ISSN 1818 -9334, PP: 821825, April 18-20, 2012.

[22] Anna Maria Masci, Jeffrey Roach, Bernard de Bono, Pierre Grenon, Lindsay Cowell, "Bridging multiple Ontologies Representation of the liver Immune Response", International Conference on Biomedical Ontologies (ICBO), Buffalo,NY, USA Working with multiple Biomedical Ontologies Workshop, 2011.

[23] NatalieBruc, GalinaMagariu, TatianaVerlan," Gallbladder Description in Ultrasound Images Ontology", Proceeding of Modelling and Development of Intelligent Systems, PP: 18-27, Sibiu-Romania, 2011.

[24] Natalia BRUC," An approach to mapping between the classification of diseases ICD-10 and the Ontology of Ultrasound Images of hepato-pancreato-biliary zone organs", 8th International Conference on Microelectronics and Computer Science, Chisinau, Republic of Moldova, PP: 314-317, 2014

[25] DICOM, Available: http://dicom.nema.org/, last visited 12 January, 2015

[26] International Statistical Classification of Diseases and Related Health Problems 10th Revision (ICD-10), Available: http://apps.who.int/classifications/icd10/browse/2010/en\#/K80K87, last visited 4 December, 2014.

[27] N. Bruc, G. Magariu, T. Verlan, "Elaborating of Ultrasound Images Ontology in Ultrasound Diagnostics", in the International Conference on e-Health and Bioengineering, EHB, Iasi, In CD Gr.T.Popa University of Medicine and Pharmacy Publishing House, Iaşi, România, Editors: Hariton Costin, Alexandru Morega, Liliana Vereștiuc. ISBN: 978-606-544-078-4, 2011

[28] Kouji Kozaki, Hiroko Kou, Yuki Yamagata, Takeshi Imai, Kazuhiko Ohe, Riichiro Mizoguchi," Browsing Casual Chain in a Disease Ontology", International Semantic Web Conference (Posters \& Demos)'12, 2012. 
[29] Kafali, Ozgur; Sindlar, Michal; Weide, Tom van der; Stathis, Kostas," ORC: an Ontology Reasoning Component for Diabetes", 2nd International Workshop on Artificial Intelligence and Netmedicine (NetMed'13). 2013.

[30] Kafal1,Ö.,Bromuri,S.,Sindlar,M.,vanderWeide,T.,Pelaez,E.A.,Scha echtle,U.,.Stathis,K, "COMMODITY12:A smarte-health environment for diabetes management", Journal of Ambient Intelligence and Smart Environments, IOS Press (Toappear), 2013.

[31] Rung-Ching Chen, Yun-Hou Huang, Cho-Tsan Bau, Shyi-Ming Chen," A recommendation system based on domain ontology and SWRL for anti-diabetic drugs selection", Expert Systems with Applications, Volume 39, Issue 4, PP: 3995-4006, 2012.
[32] Lasierra N , Alesanco A, Guillén S, García J, "A three stage ontology-driven solution to provide personalized care to chronic Patients at home", Journal of Biomedical Informatics, PP: 516529, 2013.

[33] David Riaño, Francis , Joan Albert López-Vallverdú, Fabio Campana, Sara Ercolani, Patrizia Mecocci,, Roberta Annicchiarico, Carlo Caltagirone," An ontology-based personalization of health-care knowledge to support clinical decisions for chronically ill patients", Journal of Biomedical Informatics Volume 45, Issue 3, PP: 429-446, 2012. 